\section{Gemeinsam für die Allerkleinsten - \\ der Bundesverband „Das frühgeborene Kind“}

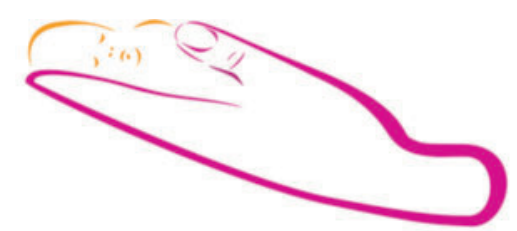

Bundesverband

"Das frühgeborene Kind" e.V.
Nicht selten endet eine Schwangerschaft vor der 40. SSW, in manchen Fällen deutlich früher. In Deutschland kommen jährlich rund 60000 Frühgeborene zur Welt. Für betroffene Eltern ändert sich von einem Tag auf den anderen das ganze Leben. Da ist Unterstützung gefragt-sowohl durch Familie und Freunde als auch von professioneller Seite. Daher wurde 1992 der Bundesverband „Das frühgeborene Kind“ e. V. gegründet.

Es schlossen sich über 80 Vertreter verschiedener Initiativen und Vereine zu einer Dachorganisation zusammen. Der gemeinnützige Verein versteht sich als zentrale Kontakt- und Vermittlungsstelle für Eltern mit einem frühgeborenen Kind. Neben dem Vorstand aus betroffenen Eltern, die ehrenamtlich arbeiten, gibt es einen wissenschaftlichen Beirat aus Fachleuten verschiedenster Berufsgruppen. In den gut 25 Jahren seines Bestehens ist der Verband zu einem professionellen Ansprechpartner für Eltern und Fachleute geworden. Finanziert werden die vielfältigen Aktivitäten durch Spenden, die Selbsthilfeförderung der Krankenkassen und Mitgliedsbeiträge. Auch Unternehmen und Stiftungen unterstützen das Engagement für die Allerkleinsten.

\section{Umfangreicher Service}

www.fruehgeborene.de - die Website des Verbands - ist mit ihrer Fülle an Tipps eine wichtige Informationsquelle-für betroffene Eltern ebenso wie für Ärzte, Therapeuten, Pädagogen und Hebammen. Für Eltern, die sich zum ersten Mal mit dem Themenkomplex beschäftigen, bietet das Glossar „Frühchen-ABC“ einen guten Einstieg. Von A wie Alveolen bis Z wie Zyanose gibt es kurze Erklärungen zu vielen Fachbegriffen, die Laien verwirren können. Zusätzlich bietet der Verband eine kostenfreie Telefon-Hotline an, die täglich erreichbar ist. Hier geben erfahrene Mütter von Frühgeborenen Rat und verweisen an andere Ansprechpartner. Ein vierteljährlich erscheinendes Verbandsmagazin mit jeweils einem Schwerpunktthema, zahlreiche Informationsbroschüren, ein Newsletter und ein Online-Shop komplettieren das Angebot.

\section{Infos für Eltern und Familie}

Die Informationsangebote richten sich zunächst an die betroffenen Eltern. Ihnen wird die Situation, in der sie sich so überraschend befinden, noch einmal erläutert und sie erhalten Empfehlungen, wie sie damit umgehen können. Enthalten sind Informationen zu Stillen und Körperkontakt zum Baby, aber auch Tipps für Organisatorisches wie notwendige Behördengänge und andere Formalitäten (z. B. Musteranträge mit Formulierungen, die Eltern übernehmen können). Zu verschiedenen medizinischen Problemen stehen Info-Briefe zum Download auf der Webseite bereit. Da Frühgeborene meist nach der Entlassung aus der Klinik besondere Bedürfnisse haben, gibt es zudem eine Liste für Kinderarztpraxen mit neonatologischem Schwerpunkt.

In einer eigenen Rubrik erhalten Großund Pflegeeltern hilfreiche Tipps. Um Geschwisterkindern die Besonderheiten eines zu früh geborenen Babys nahezubringen, bietet der Verband seit Herbst 2017 die App „Hallo Frühchen“ an. Sie richtet sich an Kinder im Vorschulalter und bietet u.a. ein interaktives Bilderbuch, Erkläranimationen, Wimmelbilder und ein interaktives Fotoalbum.

\section{Infos für Fachleute}

Der Bundesverband „Das frühgeborene Kind“ macht es sich zur Aufgabe, mit Fachleuten stets im Dialog zu sein. Dazu bietet er Fortbildungen an und stellt Referenten zur Verfügung. So bietet der Verband gemeinsam mit der Deutschen Gesellschaft für Neonatologie und
Pädiatrische Intensivmedizin (GNPI) seit 2016 eine Weiterbildung für Fachkräfte an, die für die psychosoziale Beratung und Begleitung von Eltern von Frühgeborenen bzw. krank geborenen Kindern in Perinatalzentren qualifiziert.

Ähnlich wie bei den Informationen für Eltern, gibt es auch für Kinderärzte, Therapeuten und pädagogische Fachkräfte eine Vielzahl von Informationsbroschüren, z. B. einen Leitfaden für den Umgang mit betroffenen Familien. Zum Thema Palliativversorgung und Trauerbegleitung hat sich eine interdisziplinäre Projektgruppe zusammengefunden, die Leitsätze als praxisnahe Orientierung für Behandlungsteams und Beratende formuliert hat. Die im 2018 erschienene Broschüre steht ebenfalls auf der Website zum Download bereit.

\section{Hilfe zur Selbsthilfe}

Für Eltern von Frühgeborenen ist sowohl die Unterstützung von Fachleuten als auch der Rat von (ehemaligen) Betroffenen wichtig. Etwa 80 Selbsthilfe-Initiativen sind daher Ansprechpartner auf lokaler Ebene und werden vom Bundesverband dabei unterstützt. In der Liste finden sich neben überregionalen Angeboten rund 80 regionale Adressen in ganz Deutschland. So finden Eltern auch vor Ort die richtigen Ansprechpartner.

KONTAKT

Bundesverband Das frühgeborene

Kind e. V.

Darmstädter Landstr. 213

60598 Frankfurt am Main

Telefon: 06958700990

E-Mail: info@fruehgeborene.de www.fruehgeborene.de 